\title{
A Compact Strain Sensor Based on Microstructural Fabry-Perot Fiber Cavity
}

\author{
Zhiwu Guo', Yannan Wang² and Jin $\mathrm{Li}^{1,2,3, *}$
}

1 State Key Laboratory of Nuclear Power Safety Monitoring Technology and Equipment, Shenzhen, Guangdong, 518172, China; guozhi.ww@163.com (Zhiwu Guo); lijin@ise.neu.edu.cn (Jin Li)

2 College of Information Science and Engineering, Northeastern University, Shenyang, 110819, China, China; 2597831105@qq.com (Yannan Wang); lijin@ise.neu.edu.cn (Jin Li)

3 Hebei Key Laboratory of Micro-Nano Precision Optical Sensing and Measurement Technology, Qinhuangdao, 066004, China; lijin@ise.neu.edu.cn (Jin Li)

* Correspondence: lijin@ise.neu.edu.cn

\begin{abstract}
Fabry-Perot air chamber was constructed at the melting point (splicing location) of two single-mode fibers by glycerin assisted self-expansion method. The morphology of the Fabry-Perot air chamber was fabricated and optimized by modulating the splicing parameters (drawing process, discharging location, time and intensity) and the fibers' end-face (plane or arc). The in-line or reflected Fabry-Perot cavities have been applied to determine the tensile strain in the range of 0-1.2 N. The train sensing performance of the spherical shaped FP cavity has been experimentally demonstrated with the best sensitivity of $3.628 \mathrm{~nm} / \mathrm{N}$, corresponding to the resolution of $\sim 0.005 \mathrm{~N}$. The proposed FP fiber sensor has the advantages of low cost, fast fabrication and easy-integration with the common fiber system.
\end{abstract}

Keywords: Optical fiber sensor; Fabry-Perot interferometer; Strain sensor

\section{Introduction}

The Fabry-Perot (FP) interferometer was invented by two French physicists named Charles Fabry and Alfred Perot in 1897, and became an important optical interference instrument in physics and engineering applications. Among all kinds of optical fiber sensors, the research of novel microstructure FP interferometer fiber sensor is favored and widely concerned by scholars at home and abroad, because of its simple structure, high sensitivity, wide sensing frequency band, no electromagnetic interference, and can work at high temperature [1,2]. Optical fiber sensor is especially suitable for the measurement of static low pressure, micro-pressure, sound wave, vibration and other physical quantities, especially in high temperature, strong magnetic field interference and flammable and explosive environment [3-5]. In addition, the miniature FP interferometric probe can form a local "point" sensor, which is conducive to meet the needs of the optical fiber sensing network for integration and miniaturization. In recent years, many scholars have proposed a variety of FP sensors with different optical fiber structure [6,7]. In 1981, S J Petuchowski et al. firstly designed the optical fiber sensor based on FP cavity [8]. Since Lee and Talyor announced the successful fabrication of optical fiber sensor with intrinsic FP interferometer structure in 1988, optical fiber FP sensor has gradually become an indispensable role in the optical fiber sensors [9].

With the continuous development of FP fiber sensor technology, it has been widely used to measure displacement, pressure, acceleration and temperature [10]. Optical fiber FP sensor has the advantages of high sensitivity, good resolution, wide dynamic response and smart structure [11]. The sensitivity has been seriously limited by the thermal optical coefficient and thermal expansion coefficient of the optical fiber material itself [12]. The FP interference cavity was usually prepared as a groove or a penetrating hole on a single 
single-mode fiber or a multi-mode fiber through high-precision laser processing or chemical etching technology $[13,14]$. In order to obtain more ideal response effect, it is a good choice to encapsulate sensitive materials in the FP cavity, where the sensing performance can be effectively optimized due to the modulation of the sensitive materials' refractive index or volume responding to the surrounding environment, such as the liquid or gas concentration, humidity, temperature and magnetic [15-18]. The film covered FP cavity can also been used to determine the voice frequency and intensity [19]. However, the FP cavities fabricated by the above methods have been seriously limited by the high fabrication cost, low structural repeatability and poor compactness.

In this paper, we proposed a FP air cavity for measuring tensile stress by fusing common single-mode fibers (SMFs) with a simple and low-cost fabrication process.

\section{Fabry-Perot Cavity Preparation}

To design the FP stress probe, two single-mode optical fibers with glycerine end face were fused together. Because glycerine vaporized during the discharge splicing process, a spherical cavity was formed between two end faces. The two-inner surface of the spherical cavity play as the two reflecting surfaces of the F-P interference cavity, respectively. When the stress was applied on the F-P cavity structure, it was lengthened. According to the sensing mechanism, the interference wavelength will be changed as well. The stress response curve was illustrated by the phase demodulation method to measure stress values. Firstly, the end face of single-mode optical fiber was pretreated, since it is too difficult to disperse the glycerin liquid uniformly on the flatted surface shown in Fig. 1(a).
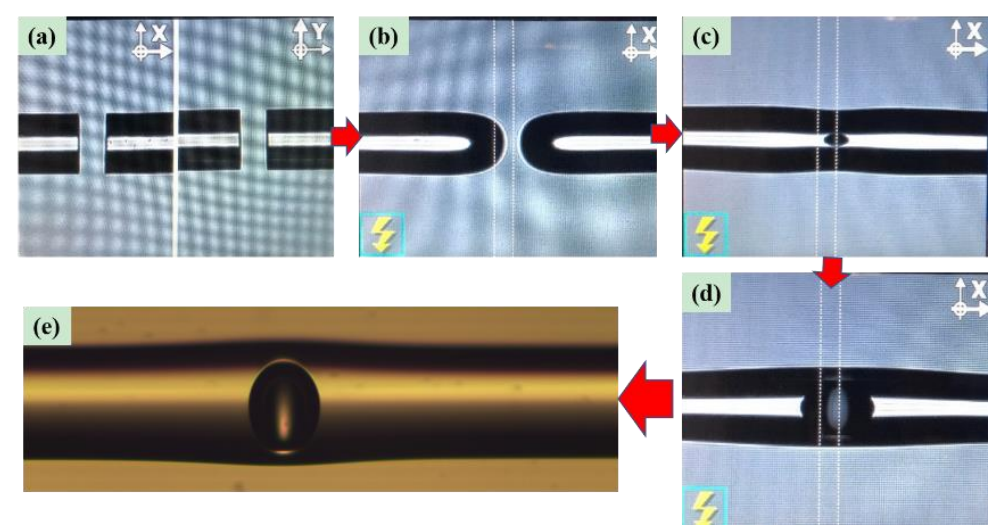

Figure 1. Comparison of SMF end face (a) before and (b) after pretreatment; Comparison of cavity (c) before and (d) after enlargement; (e) Micrograph of FP fiber optic stress sensor.

The end face of optical fiber was melted and deformed by the instantaneous high temperature from the arc discharge with the during time of $1200 \mathrm{~ms}$ and intensity of 100 . During the cooling process, the end face became radian shape (as illustrated in Fig. 1(b)) due to its own surface tension. After glycerin was dipped onto the radian end face, a slight squeezing force was generated between the two optical fibers by manually controlling the motor. Due to the large curvature, the glycerin liquid can be gathered in the middle region to form an oil film after contact, instead of diffusing to both sides. The initial cavity with small volume was formed after discharge, as shown in Fig. 1(c). Appling the secondary arc discharge and the tensile prestress action, the residual glycerin continues to vaporize, thus increasing the cavity volume, becoming larger than the diameter of the ordinary single-mode fiber of $125 \mu \mathrm{m}$. The enlarged cavity and the finally formed F-P cavity fabricated are shown in Fig. 1(d) and Fig. 1(e), respectively. 


\section{Results}

The schematic diagram of stress sensing experimental system based on FP cavity optical fiber stress sensor is shown in Fig.2 below.

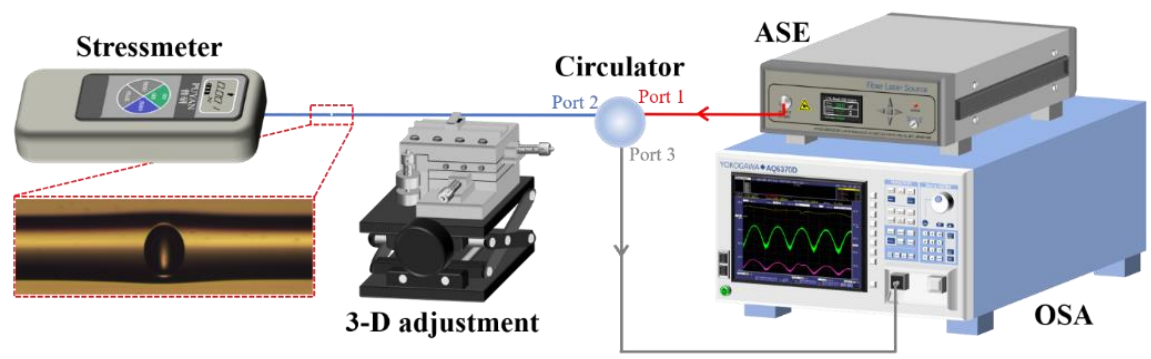

Figure 2. Schematic diagram of stress sensing experimental system.

The experimental system includes amplified spontaneous emission (ASE) light source, optical fiber circulator, precision 3-D adjustment platform, stress meter and optical spectrum analyzer (OSA, resolution: $20 \mathrm{pm}$ ). The light beam is emitted from the light source and enters into the stress sensor through the Port1 and Port 2 of the circulator. After being reflected by the FP air-cavity, the signal light is collected by the spectrometer through port3 of the circulator. The optical fiber stress sensor is formed by fusing two SMFs, one of which is connected with the circulator Port2 and fixed on to a 3-D adjustment. The other SMF is connected to the stress meter. By manually adjusting the 3-D adjustment to supply stress along the fiber structure, the FP cavity length was changed, resulting in the movement of the resonance wavelength of the interference spectrum on spectrometer. The position of interference wavelength can be obtained by single peak demodulation method, and the relationship between the shift of interference wavelength and the change of stress can be established, so as to realize the stress detection.

According to the above experimental sensing system and experimental process, the stress sensing experiment of spherical cavity FP optical fiber stress sensor with the transverse diameter of $77 \mu \mathrm{m}$ and longitudinal diameter of $104 \mu \mathrm{m}$ is carried out. When the stress increases from $0 \mathrm{~N}$ to $1.2 \mathrm{~N}$, the spectrum shifts to red due to the length stretching of the F-P interference cavity. The movement of the interference spectrum in the whole spectrum range of the light source is shown in Fig. 3.

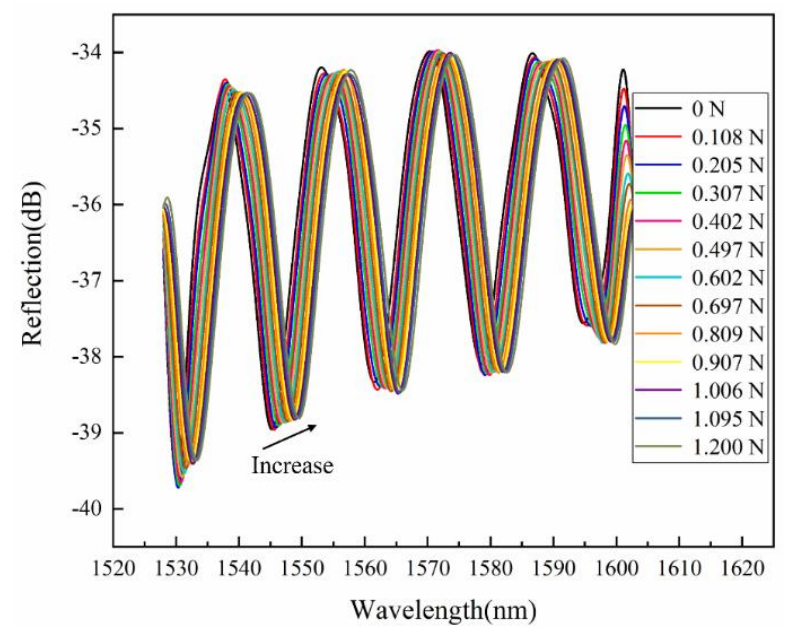

Figure 3. Comparison of SMF end face (a) before and (b) after pretreatment; Comparison of cavity (c) before and (d) after enlargement; (e) Micrograph of FP fiber optic stress sensor.

The free spectrum range (FSR) for the FP interference spectrum can be represented by 


$$
F S R=\frac{\lambda^{2}}{2 n L}
$$

$\lambda$ refers to the center working wavelength of the light source $1550 \mathrm{~nm}, \mathrm{n}$ is the refractive index of air 1 . The FSR can be calculated as

$$
F S R=\frac{1550^{2} \mathrm{~nm}^{2}}{2 \times 1 \times 77000 \mathrm{~nm}} \approx 15.6 \mathrm{~nm}
$$

This value is smaller compared to the FSR of $\sim 16.29 \mathrm{~nm}$ in the spectrum of Fig. 3, which is caused by the little offset of the FP cavity from the central axis of the optical fiber core. The offset issue will be further discussed in Section 4. In this experiment, the stress exerted on the FP cavity along the optical fiber can be determined by tracing the wavelength locations of one special dip in the spectra of Fig. 3. A regular red shift has been observed in the interference spectrum, as illustrated in Fig. 4.
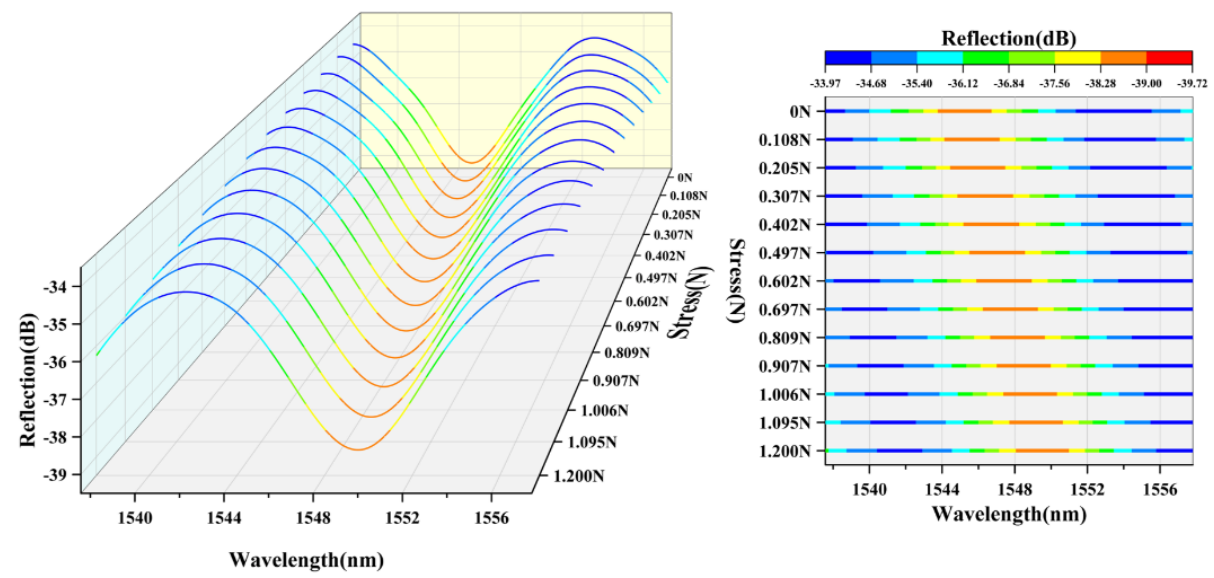

Figure 4. Special dip movement with exerted stress.

The fitting stress sensing characteristic curve is shown in Fig. 5. The experimental results indicate a stress sensitivity of $3.624 \mathrm{~nm} / \mathrm{N}$ and a resolution of $0.005 \mathrm{~N}(20 \mathrm{pm} / 3.624$ $\mathrm{nm} / \mathrm{N})$.

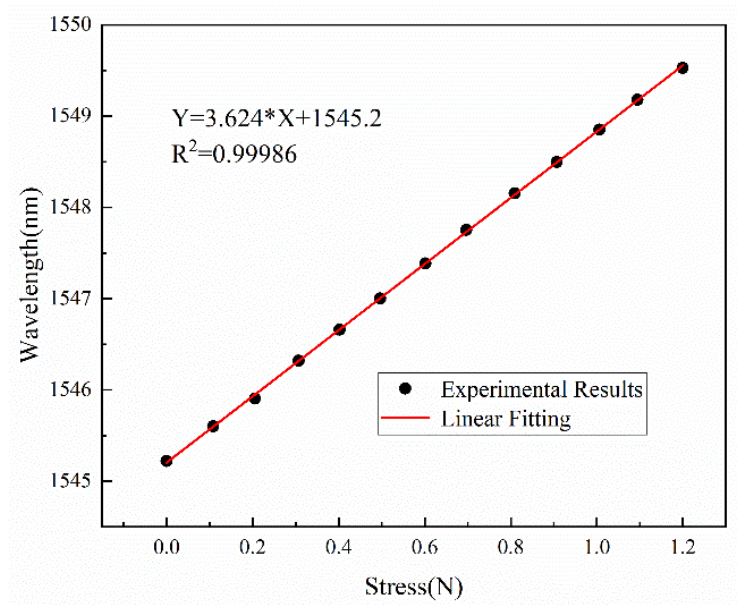

Figure 5. Stress sensing characteristic curve as stress rises from $0 \mathrm{~N}$ to $1.2 \mathrm{~N}$.

In addition to the transverse diameter of $77 \mu \mathrm{m}$ and longitudinal diameter of $104 \mu \mathrm{m}$, the fiber diameter near the FP cavity is $\sim 138 \mu \mathrm{m}$. Because the applied stress direction is 
parallel to the optical fiber and perpendicular to the central section of the cavity, the stress area of the cavity sensing structure is an annular area

$$
A=\pi R^{2}-\pi r^{2} \approx 0.0064623 \mathrm{~mm}^{2}
$$

Where, $R$ and $r$ are $69 \mu \mathrm{m}$ and $52 \mu \mathrm{m}$, respectively, corresponding to the fiber radius at the cavity center and the longitudinal radius of the cavity. According to Hooke's law, the positive strain in the elastic range is

$$
\varepsilon=\frac{\sigma}{E}=\frac{F}{A E}=\frac{0.1 \mathrm{~N}}{6.4623 \times 10^{-9} \mathrm{~m}^{2} \times 72 \times 10^{9} \mathrm{~Pa}} \approx 2.149 \times 10^{-4}
$$

Here, $\sigma, F, A, \varepsilon$ refer to positive stress, force, effect area and positive strain, respectively. Fiber material is silica with the Young's modulus of $72000 \mathrm{MPa}$. The positive strain is also the relative variation, that is, the ratio of the stress variable of the length of FP interference cavity to its original length before the stress being exerted, so it can be concluded that under the stress of $0.1 \mathrm{~N}$, the stress variable of the FP cavity is

$$
\Delta L=\varepsilon L=2.149 \times 10^{-4} \times 77 \mu \mathrm{m} \approx 0.0165 \mu \mathrm{m}
$$

Here, $\lambda$ When the length of FP interference cavity increases, the interference wavelength increases, resulting in red-shift. Using central wavelength $\lambda$, the interference grade can be calculated as

$$
m=\frac{2 n L}{\lambda}=\frac{2 \times 1 \times 77 \mu \mathrm{m}}{1.55 \mu \mathrm{m}} \approx 99
$$

Under the stress of $0.1 \mathrm{~N}$, the red shift of the interference spectrum is

$$
\Delta \lambda=\frac{2 n}{m} \Delta L=\frac{2 \times 1}{99} \times 16.5 \mathrm{~nm}=0.3333 \mathrm{~nm}
$$

Therefore, there is a small error between the theoretical sensitivity value of 3.333 $\mathrm{nm} / \mathrm{N}$ and the experimental value of $3.624 \mathrm{~nm} / \mathrm{N}$, which is mainly caused by the measurement error of the sensing structure and the small axial offset of the FP cavity. The experimental results show that the preparation of the sensing structure and the stress sensing performance are very good within the allowable error range.

The stress sensing characteristic curve was obtained by repeating the increasing and decreasing stress experiment, as the red fitting curve shown in Fig. 6. The black (circular) and red (pentagonal) experimental data points and the corresponding linear fitting lines respectively represent the stress response results of the of stress increase and decrease process. It is obvious that the sensitivity during the stress increase process $(3.624 \mathrm{~nm} / \mathrm{N})$ and the stress decrease process $(3.628 \mathrm{~nm} / \mathrm{N})$ are close to each other, indicating the good stability of the sensor. The two fitting lines are not completely coincident with the overall translation of $\sim 0.2 \mathrm{~nm}$, which is mainly due to the slight sliding of the fiber system, as well as the interference wavelength drift during the stress transition from the increase to decrease process. 


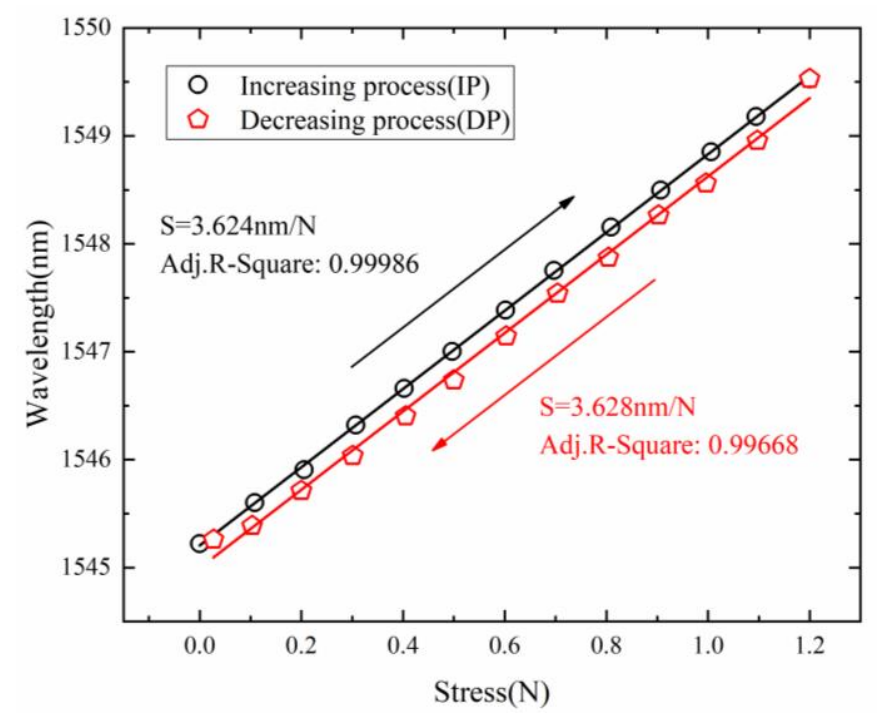

Figure 6. Stress sensing characteristic curve.

The optical structure was more firmly fixed on the 3-D adjustment to reduce the influence of sliding effect. The stress sensing experiment was carried out for the fiber FP spherical cavity with a transverse diameter of $73 \mu \mathrm{m}$, longitudinal diameter of 97 and optical fiber diameter of $140 \mu \mathrm{m}$. The step stress value was still $0.1 \mathrm{~N}$. The final sensitivity characteristic curve is shown in Fig. 7.

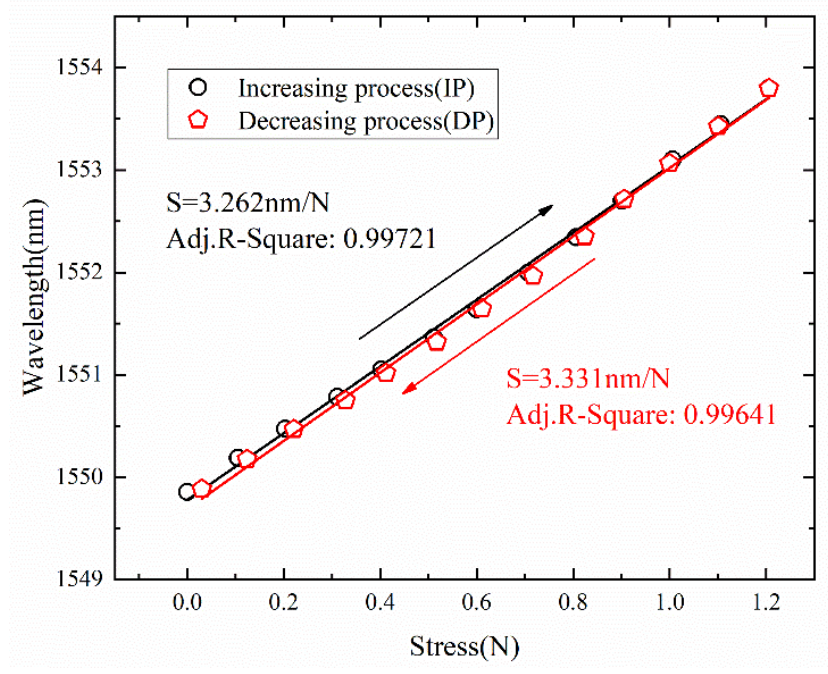

Figure 7. Stress sensing characteristic curve of FP cavity structure with a transverse diameter $73 \mu \mathrm{m}$ and a longitudinal diameter $97 \mu \mathrm{m}$.

The sensitivity values are $3.262 \mathrm{~nm} / \mathrm{N}$ and $3.331 \mathrm{~nm} / \mathrm{N}$ during the stress increase and decrease process, respectively. The stress resolution is $\sim 0.006 \mathrm{~N}$. The coincidence degree of sensitivity fitting line has been obviously improved compared to the previous experment. In addition, the maximum sensitivity of $3.331 \mathrm{~nm} / \mathrm{N}$ is less than that of the previous structure $(3.624 \mathrm{~nm} / \mathrm{N})$. The main reason is that the stress cross-sectional area becomes larger due to the bigger volume of the solid silica outside the cavity (inner air diameter 97 $\mu \mathrm{m}<104 \mu \mathrm{m}$, outer silica diameter $140 \mu \mathrm{m}>138 \mu \mathrm{m}$ ). 


\section{Discussion}

To study the limit of this stress fiber sensor, a FP structure with a transverse diameter of $85 \mu \mathrm{m}$ and a longitudinal diameter of $116 \mu \mathrm{m}$ was fabricated. The spectral shift diagram obtained by increasing the stress with the step value of $0.3 \mathrm{~N}$ is shown in Fig. 8.

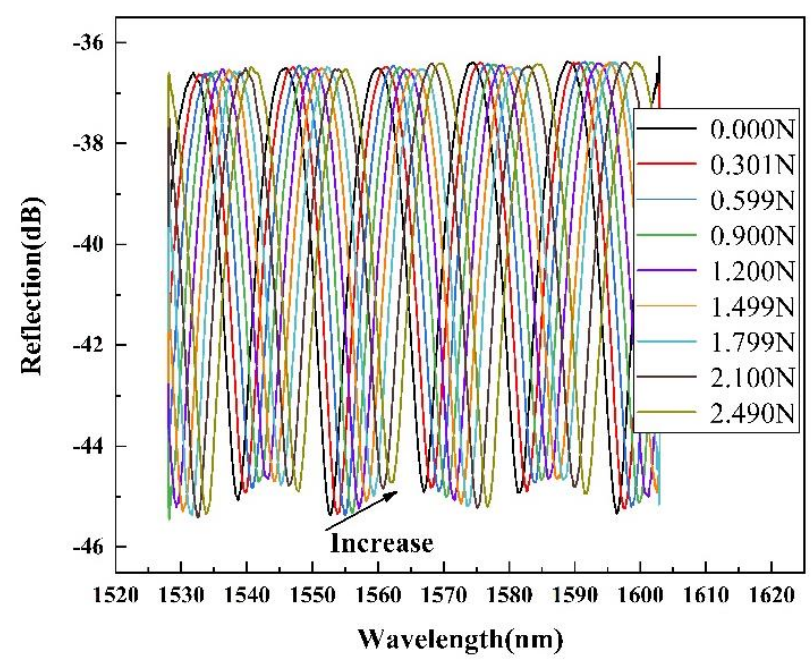

Figure 8. Spectrum of stress limit test of sensing structure with a transverse diameter $85 \mu \mathrm{m}$ and a longitudinal diameter $116 \mu \mathrm{m}$.

The FSR of the spectrum is $\sim 14 \mathrm{~nm}$, which is basically equal to the theoretical value of $14.1 \mathrm{~nm}$ calculated by Eq. (1). When the applied stress increases from $0 \mathrm{~N}$ to $2.49 \mathrm{~N}$, the stable output spectrum was obtained, indicating that the FP cavity structure was not damaged. However, when the stress of $2.49 \mathrm{~N}$ was continuously applied, the spectrum disappeared and the structure was damaged as well. In addition, the red shift of the spectrum is less than the FSR, so it will be convenient to complete the demodulation process in the whole working range. The stress measurement limit of the sensing structure depends on the volume of silica surrounding the FP cavity. The stress limit can be significantly improved for a larger volume, but the sensitivity will be reduced at the same time. Therefore, the working range and sensitivity should be balanced according to the application requirements.

In the manufacturing process of stress fiber sensor, the process parameters may also lead to the position deviation of the FP cavity, and then affect the interference spectrum. A stress sensing structure with a transverse diameter of $85 \mu \mathrm{m}$ and a longitudinal diameter of $119 \mu \mathrm{m}$ was fabricated, and its interference spectrum is shown in Fig. 9 (a).
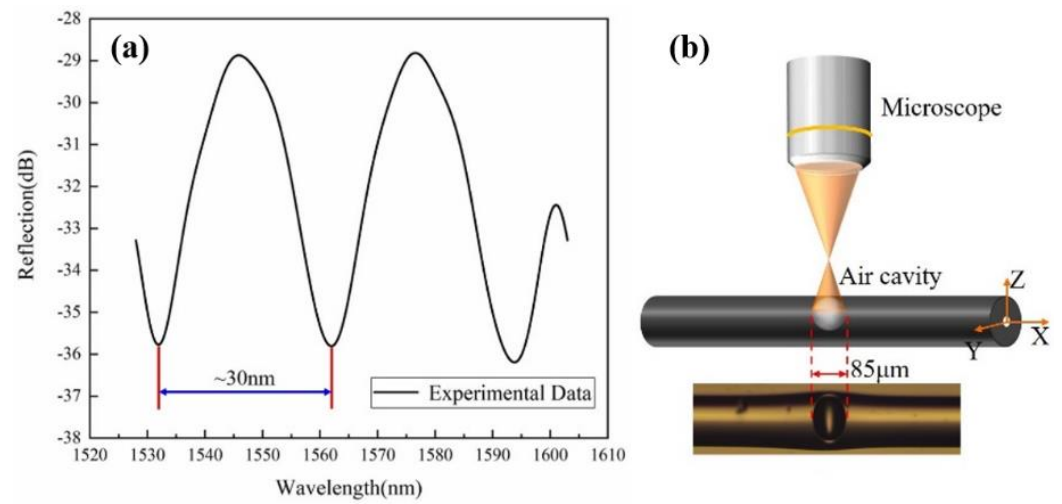

Figure 9. Sensing structure with the cavity dislocation.

The FSR is $\sim 30 \mathrm{~nm}$, which is more than two folders of the theoretical value of $14.1 \mathrm{~nm}$, that is, the FP cavity length is less than the half of the theoretical length, indicating that 
the cavity position of the structure is seriously shifted. It is confirmed in the microscope that this cavity has no offset in the $X Y$ plane, but has a serious offset in the $X Z$ plane, as shown in Fig. 9 (b).

\section{Conclusions}

In this paper, the Fabry-Perot fiber stress sensor was designed and fabricated by a spherical structure with a transverse diameter of $77 \mu \mathrm{m}$ and longitudinal diameter of 104 $\mu \mathrm{m}$. The corresponding stress sensing sensitivity is up to $3.628 \mathrm{~nm} / \mathrm{N}$ with a good repeatability and stable structure. It is approximately equal to the theoretical value. By improving the experimental conditions, the stress sensing experiment of spherical FP cavity with transverse diameter of $73 \mu \mathrm{m}$ and longitudinal diameter of $97 \mu \mathrm{m}$ is carried out. It is concluded that the maximum stress sensitivity is $3.331 \mathrm{~nm} / \mathrm{N}$. The stress detection limit of $2.49 \mathrm{~N}$ has been experimentally demonstrated by using one fiber FP cavity with transverse diameter of $85 \mu \mathrm{m}$ and longitudinal diameter of $116 \mu \mathrm{m}$. The designed compact FP stress probe is expected to be integrated into the wearable system for real-time monitoring of life status.

Author Contributions: Investigation, data curation, writing - original draft preparation, Z.W. Guo; writing-review and editing, Y.N. Wang; supervision, project administration, J. Li; funding acquisition, Y.Y. All authors have read and agreed to the published version of the manuscript.

Funding: This research was funded by the Fundamental Research Funds for Central Universities (N2004007), and Hebei Natural Science Foundation (F2020501040).

Institutional Review Board Statement: Not applicable.

Informed Consent Statement: Not applicable.

Conflicts of Interest: The authors declare no conflict of interest.

\section{References}

1. Ran, Z.L.; He, X.; Rao, Y.J.; Sun, D.; Qin, X.J.; Zeng, D.B.; Chu W.W.; Li X.K.; Wei, Y.B. Fiber-Optic Microstructure Sensors: A Review. Photonic Sensors 2021, 11(2), pp. 227-261.

2. Hosseinzadeh Sani, M.; Ghanbari, A.; Saghaei, H. An ultra-narrowband all-optical filter based on the resonant cavities in rodbased photonic crystal microstructure. Optical and Quantum Electronics 2020, 52, pp. 1-15.

3. Budinski, V.; Donlagic, D. A Miniature Fabry Perot Sensor for Twist/Rotation, Strain and Temperature Measurements Based on a Four-Core Fiber. Sensors 2019, 19(7), pp. 1574.

4. Wang, X.; Wang, Q.; Song, Z.; Qi, K. Simulation of a microstructure fiber pressure sensor based on lossy mode resonance. AIP Advances 2019, 9(9), pp. 095005.

5. Tang, S.; Zhang, B.; Li, Z.; Dai, J.; Wang, G.; Yang, M. Self-compensated microstructure fiber optic sensor to detect high hydrogen concentration. Optics Express 2015, 23(17), pp. 22826-22835.

6. Zhu, J.; Wang, M.; Chen, L.; Ni, X.; Ni, H. An optical fiber Fabry-Perot pressure sensor using corrugated diaphragm and angle polished fiber. Optical Fiber Technology 2017., 34, pp. 42-46.

7. Li, X.; Chen, N.; Zhou, X.; Gong, P.; Wang, S.; Zhang, Y.; Zhao, Y. A review of specialty fiber biosensors based on interferometer configuration. Journal of Biophotonics 2021, pp. e202100068.

8. Tan, X.; Li, M.; Arsad, N.; Wen, X.; Lu, H.; An effective temperature compensation approach for ultrasonic hydrogen sensors, Review of Scientific Instrumentation 2018, 89(3), pp. 035005.

9. Gibbs, H.M.; Mccall, S.L.; Venkatesan, T.N.C.; Differential gain and bistability using a sodium-filled Fabry-Perot interferometer, Physics Reviews Letters 1976, 36(19), pp. 1135-1138.

10. Rao Y.J.; Ran Z.L.; Gong Y. Fiber-Optic Fabry-Perot Sensors: An Introduction. CRC Press: Los Angeles, 2017.

11. Nowakowski B.K.; Smith D.T.; Smith S.T. Highly compact fiber Fabry-Perot interferometer: A new instrument design[J]. Review of Scientific Instruments 2016, 87(11), pp. 115102.

12. Li J.; Yan H.; Dang H.; Meng F.L. Structure design and application of hollow core microstructured optical fiber gas sensor: A review[J]. Optics \& Laser Technology 2021, 135, pp. 106658.

13. Paixão T.; Araújo F.; Antunes P. Highly sensitive fiber optic temperature and strain sensor based on an intrinsic Fabry-Perot interferometer fabricated by a femtosecond laser[J]. Optics Letters 2019, 44(19), pp. 4833-4836.

14. Zhang, W.; Zhuang, W.; Dong, M.; Zhu, L.; Meng, F. Dual-parameter optical fiber sensor for temperature and pressure discrimination featuring cascaded tapered-FBG and ball-EFPI. IEEE Sensors Journal 2019, 19(14), pp. 5645-5652.

15. Li, M.; Liu, Y.; Gao, R.; Li, Y.; Zhao, X.; Qu, S. Ultracompact fiber sensor tip based on liquid polymer-filled Fabry-Perot cavity with high temperature sensitivity. Sensors and Actuators B: Chemical 2016, 233, pp. 496-501. 
16. Siyu, E.; Zhang, Y. N.; Wang, X.; Zhang, Y.; Han, B. Capillary encapsulated reflective fiber optic SPR temperature sensor. Physica Scripta 2019, 94(4), pp. 045504.

17. Liu, C.; Shen, T.; Wu, H.B.; Feng, Y.; Chen, J.J. Applications of magneto-strictive, magneto-optical, magnetic fluid materials in optical fiber current sensors and optical fiber magnetic field sensors: A review. Optical Fiber Technology 2021, 65, pp. 102634.

18. Zhao, Y.; Wang, X.X.; Lv, R.Q.; Zheng, H.K.; Zhou, Y.F.; Chen, M.Q. Reflective Highly Sensitive Fabry-Perot Magnetic Field Sensor Based on Magneto-Volume Effect of Magnetic Fluid. IEEE Transactions on Instrumentation and Measurement 2021, 70, pp. 1-6.

19. Li, C.; Yu, X.; Zhou, W.; Cui, Y.; Liu, J.; Fan, S. Ultrafast miniature fiber-tip Fabry-Perot humidity sensor with thin graphene oxide diaphragm. Optics Letters 2018, 43(19), pp. 4719-4722. 